\title{
Amide III SFG Signals as Sensitive Probe of Protein Folding at Cell Membrane Surface
}

\author{
Jiahuang Huang, ${ }^{1,2}$ Kangzhen Tian, ${ }^{1,2}$ Shuji Ye, ${ }^{1,2, *}$ Yi Luo ${ }^{1,2}$ \\ ${ }^{1}$ Hefei National Laboratory for Physical Sciences at the Microscale, and Department of \\ Chemical Physics, University of Science and Technology of China, Hefei, Anhui 230026, \\ China, \\ ${ }^{2}$ Synergetic Innovation Center of Quantum Information \& Quantum Physics, University of \\ Science and Technology of China, Hefei, Anhui 230026, China
}

\section{The cleaning of $\mathrm{CaF}_{2}$ prism and bilayer preparation}

$\mathrm{CaF}_{2}$ prisms were thoroughly cleaned using a procedure with several steps: They were first soaked in toluene for at least $24 \mathrm{~h}$ and then sonicated in soap detergent solution for $0.5 \mathrm{~h}$. After that, they were rinsed with deionized (DI) water before soaking in methanol for 10 min. All of the prisms were then rinsed thoroughly with an ample amount of DI water and cleaned inside Harrick plasma chamber for 10 min immediately before depositing lipid molecules on them. Substrates were tested using SFG and no signal from contamination was detected.

Single lipid bilayers were prepared on $\mathrm{CaF}_{2}$ substrates using Langmuir-Blodgett and Langmuir-Schaefer (LB/LS) methods with a KSV mini trough LB system. The detailed procedure was similar to previous reports. ${ }^{1,2}$ The bilayers were immersed in pure water inside a $2 \mathrm{~mL}$ reservoir throughout the entire experiments.

\section{The method to enhance amide III signals}

To obtain amide III signals, several developed technical procedures were employed to significantly enhance the hardly-measureable-SFG signals in the amide III region. The key technical procedures include: (1) the adoption of a near-total-internal-reflection geometry (Figure S1); (2) the employment of higher voltage to the detector: the high voltage applied to the detector of PMT was $1100 \mathrm{~V}$ for amide III signals and $700 \mathrm{~V}$ for amide I signals. The SFG 
intensity collected with the voltage of $1100 \mathrm{~V}$ is about 25 times higher than that of $700 \mathrm{~V}$; (3) the equipping of a new and longer difference-frequency generation (DFG) crystal $\left(\mathrm{AgGaS}_{2}\right.$ crystals, $8 \times 12 \times 14 \mathrm{~mm}$ ) in $\mathrm{OPG} / \mathrm{OPA} / \mathrm{DFG}$ system (optical parametric generation/ amplification and difference frequency system based on $\mathrm{LBO}$ and $\mathrm{AgGaS}_{2}$ crystals) of commercial (EKSPLA) SFG system to generate a stable IR pulse with the energy higher than $20 \mu \mathrm{J}$. These useful procedures have made it possible to probe the very weak amide III signals.

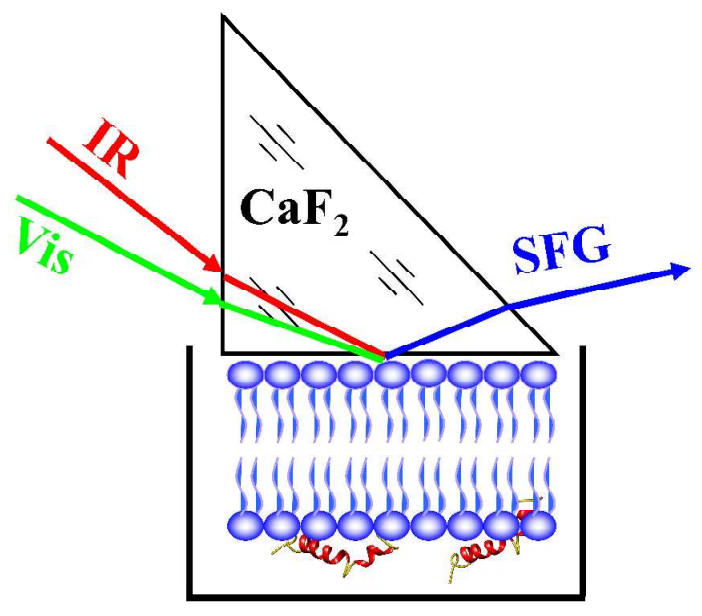

Figure S1. A near-total-internal-reflection geometry.

\section{Fitting of SFG-VS Signal}

As described in detail elsewhere, the intensity of the SFG light is related to the square of the sample's second-order nonlinear susceptibility $\left(\chi_{\text {eff }}^{(2)}\right)$, and the intensity of the two input fields $I\left(\omega_{I R}\right)$ and $I\left(\omega_{v i s}\right)$, see eq. (S1), which vanishes when a material has inversion symmetry. ${ }^{3-7}$

$I\left(\omega_{S F G}\right) \propto\left|\chi_{\text {eff }}^{(2)}\right|^{2} I_{1}\left(\omega_{v i s}\right) I_{2}\left(\omega_{I R}\right)$

where $\omega_{S F G}=\omega_{I R}+\omega_{v i s}$. As the IR beam frequency is tuned over the vibrational resonance of surface/interface molecules, the effective surface nonlinear susceptibility $\chi_{R}^{(2)}$ can be enhanced. The frequency dependence of $\chi_{\text {eff }}^{(2)}$ is described by eq. (S2).

$\chi_{e f f}^{(2)}(\omega)=\chi_{N R}^{(2)}+\sum_{v} \frac{A_{v}}{\omega-\omega_{v}+i \Gamma_{v}}$

where $A_{v}, \omega_{v}$, and $\Gamma_{v}$ are the strength, resonant frequency, and damping coefficient of the vibrational mode $(v)$, respectively. $A_{v}$ could be either positive or negative depending on the 
phase of the vibrational mode. The plot of SFG signal vs. the IR input frequency shows a polarized vibrational spectrum of the molecules at surface or interface. $A_{v}, \omega_{v}$, and $\Gamma_{v}$ can be extracted by fitting the spectrum.

\section{The SFG spectra collected at different time after pardaxin interacts with lipid bilayer}
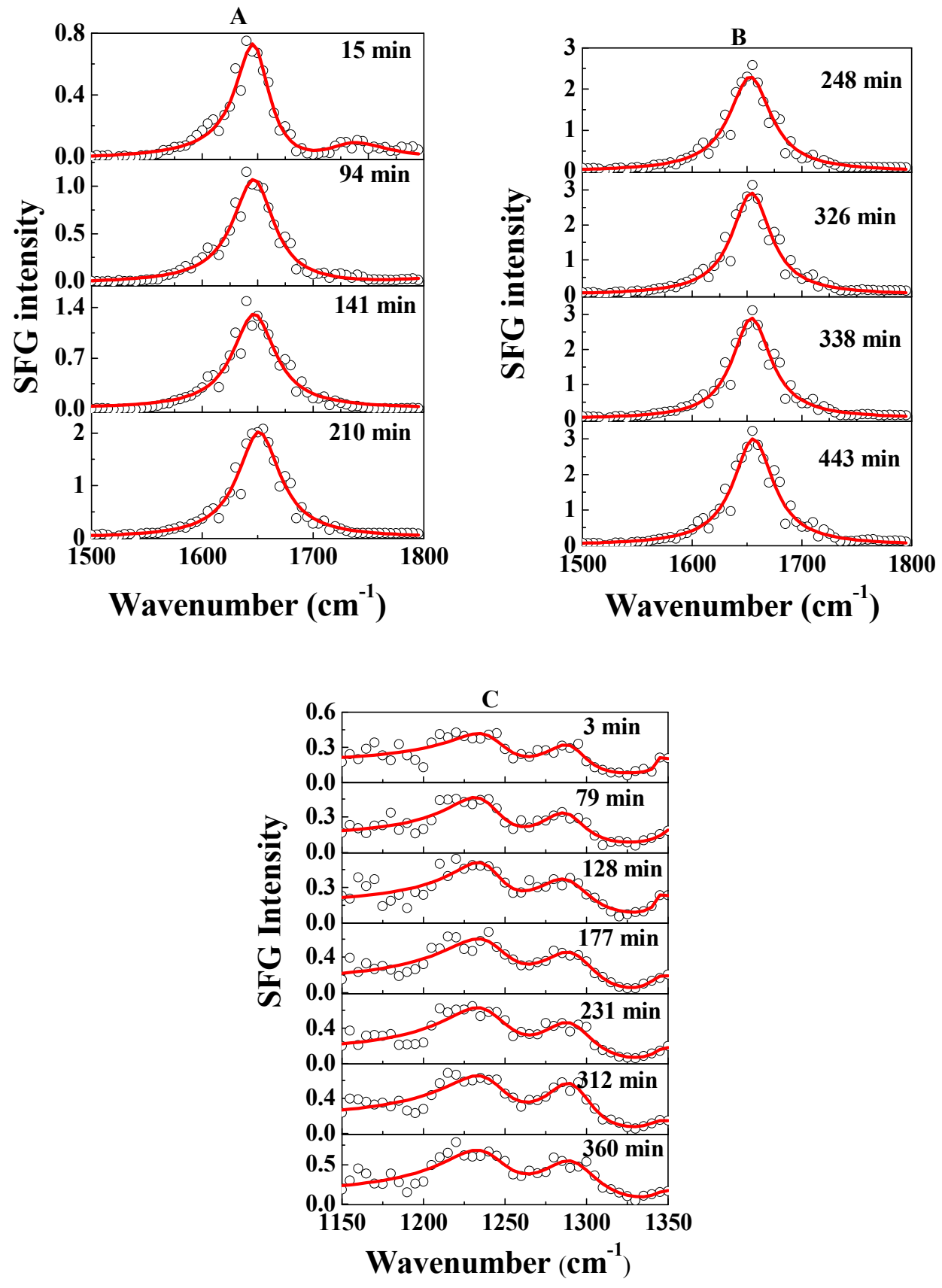

Figure S2. The ssp SFG spectra collected at different time after pardaxin interacts with dDMPC/d-DMPC lipid bilayer. A) and B) The amide I signal; C) The amide III signal. 

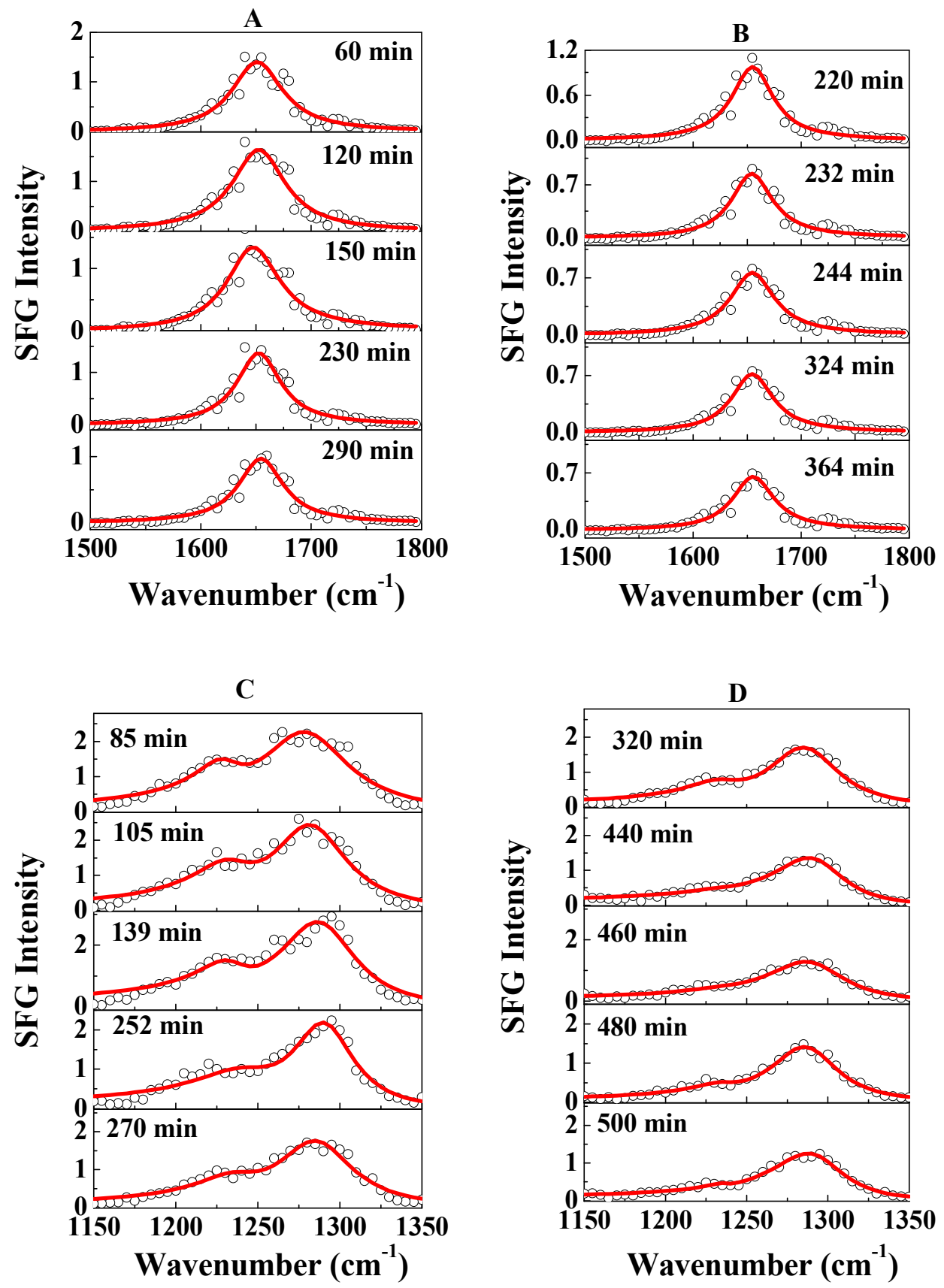

Figure S3. The ssp SFG spectra collected at different time after pardaxin interacts with dDMPG/d-DMPG lipid bilayer. A) and B) The amide I signal; C) and D) The amide III signal. 

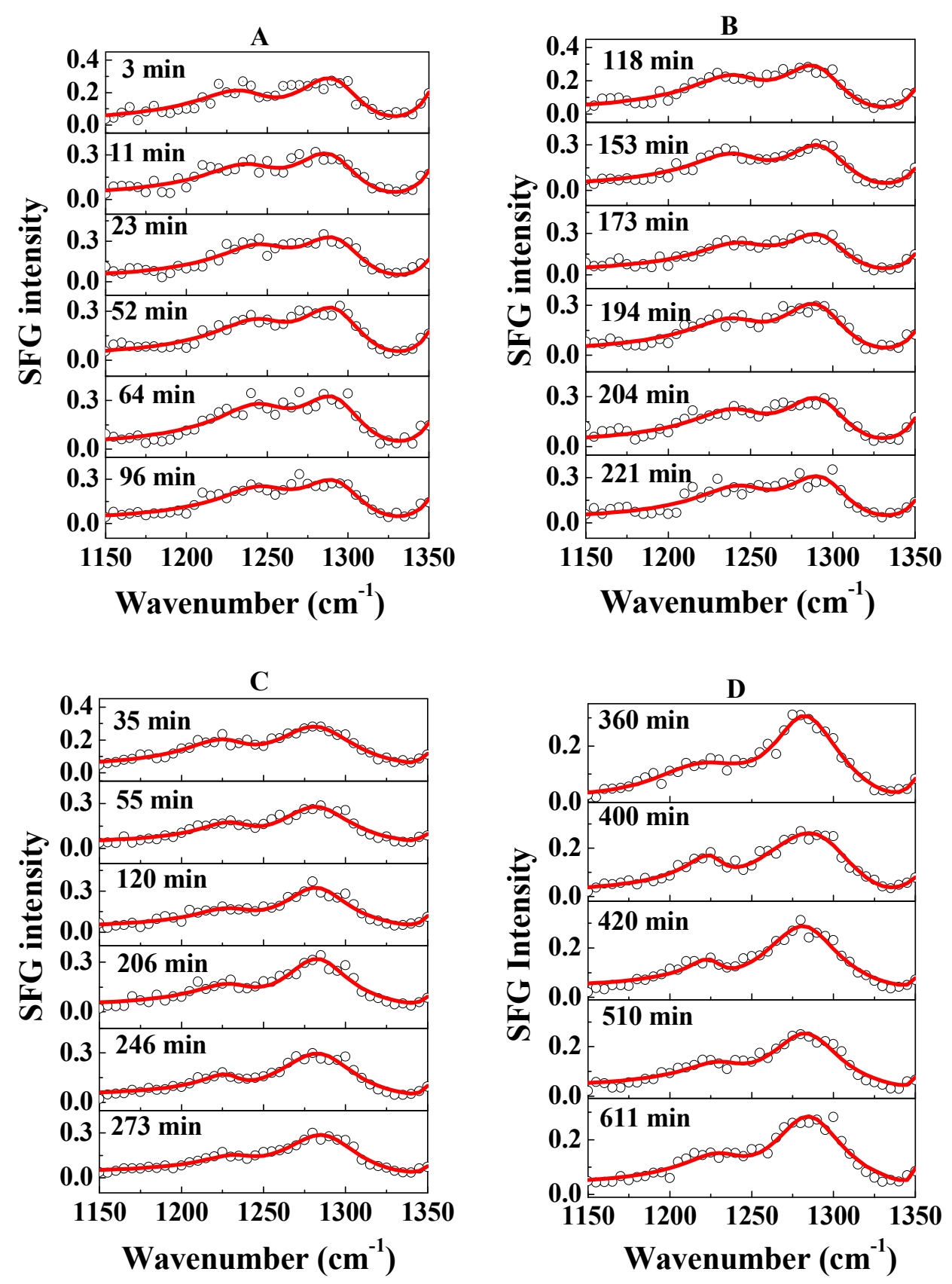

Figure S4. The ssp SFG spectra in the amide III region collected at different time after pardaxin interacts with the mixed d-DMPC and d-DMPG lipid bilayer. A) and B) d-DMPC/dDMPG (PC/PG $=4 / 1) ; C)$ and D). $d-D M P C / d-D M P G ~(P C / P G=1 / 1)$. 

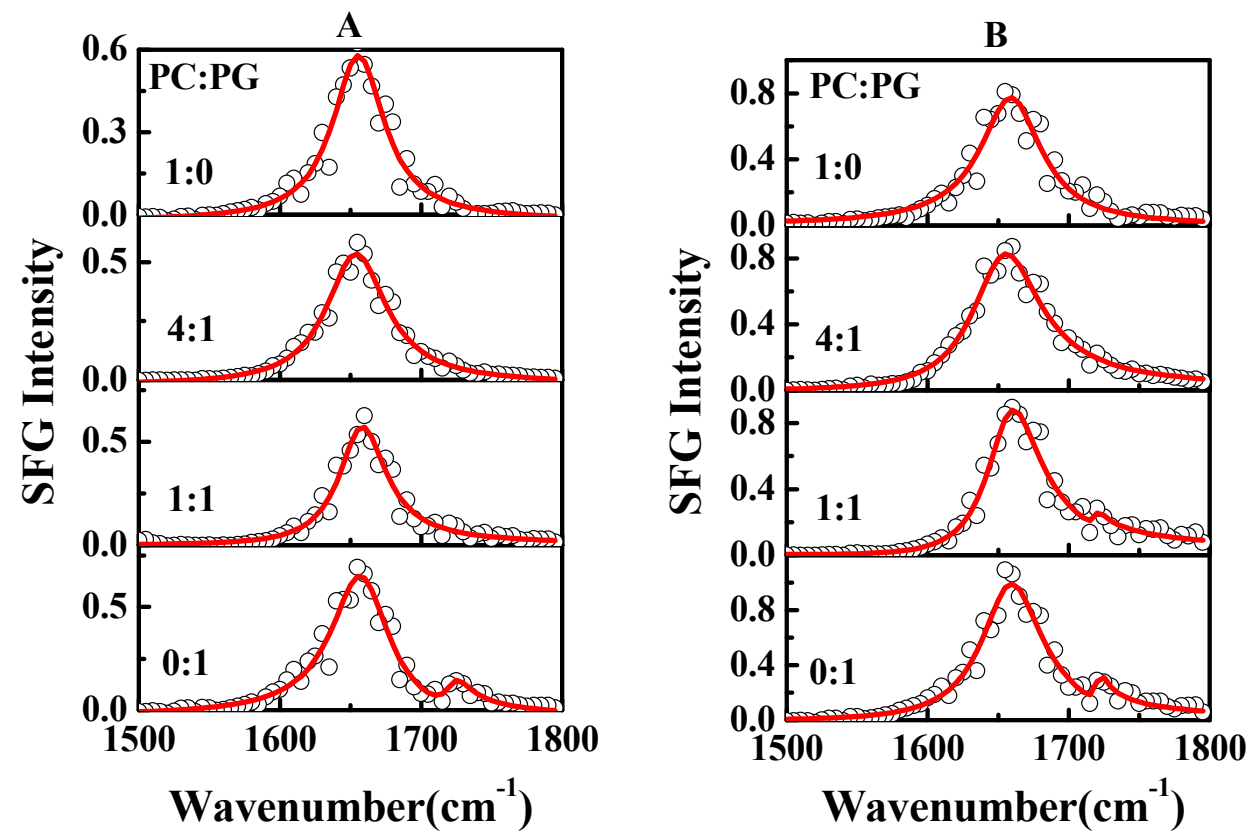

Figure S5. The amide I SFG spectra of pardaxin after the interaction between pardaxin and different lipid bilayer reached equilibrium. A) ssp; B).ppp.

\section{Reference}

(1) Ye, S. J.; Li, H. C.; Wei, F.; Jasensky, J.; Boughton, A. P.; Yang, P.; Chen, Z. Observing a Model Ion Channel Gating Action in Model Cell Membranes in Real Time in Situ: Membrane Potential Change Induced Alamethicin Orientation Change. J. Am. Chem. Soc. 2012, 134, 6237-6243.

(2) Li, H. C.; Ye, S. J.; Wei, F.; Ma, S. L.; Luo, Y. In Situ Molecular-Level Insights into the Interfacial Structure Changes of Membrane-Associated Prion Protein Fragment [118-135] Investigated by Sum Frequency Generation Vibrational Spectroscopy. Langmuir 2012, 28, 16979-16988.

(3) Shen, Y. R. The Principles of Nonlinear Optics. Wiley: New York, 1984.

(4) Castellana, E. T.; Cremer, P. S. Solid Supported Lipid Bilayers: From Biophysical Studies to Sensor Design. Surf. Sci. Rep. 2006, 61, 429-444.

(5) Lambert, A. G.; Davies, P. B.; Neivandt, D. J. Implementing the Theory of Sum Frequency Generation Vibrational Spectroscopy: A Tutorial Review. Appl. Spectrosc. Rev. 2005, 40, 103-145.

(6) Gopalakrishnan, S.; Liu, D.; Allen, H. C.; Kuo, M.; Shultz, M. J. Vibrational 
Spectroscopic Studies of Aqueous Interfaces: Salts, Acids, Bases, and Nanodrops. Chem. Rev. 2006, 106, 1155-1175.

(7) Wang, H.-F.; Gan, W.; Lu, R.; Rao, Y.; Wu, B.-H. Quantitative Spectral and Orientational Analysis in Surface Sum Frequency Generation Vibrational Spectroscopy (SFG-VS). Int. Rev. Phys. Chem. 2005, 24, 191-256. 